\title{
Laminin Modulates the Stem Cell Population in LM05-E Murine Breast Cancer Cells through the Activation of the MAPK/ERK Pathway
}

\author{
Damián E. Berardi, $\mathrm{PhD}$ \\ Diego Raffo, $\mathrm{PhD}^{1}$ \\ Laura B. Todaro, $\mathrm{PhD}^{1,2}$ \\ Marina Simian, $\mathrm{PhD}^{2,3}$
}

Correspondence: Marina Simian, $\mathrm{PhD}$ Instituto de Nanosistemas, Universidad Nacional de San Martín, Campus Miguelete, Francia y 25 de Mayo Francia, San Martín (1650),

Provincia de Buenos Aires, Argentina Tel: 54-911-5385-6555

E-mail: marina.simian@galuzzi.com

Received August 14, 2016

Accepted November 16, 2016

Published Online December 6, 2016

*Damián E. Berardi and Diego Raffo contributed equally to this work.

\begin{abstract}
Purpose
We investigated the effects of laminin on the fraction of cells with self-renewing capacity in the estrogen-dependent, tamoxifen-sensitive LMO5-E breast cancer cell line. We also determined whether laminin affected the response to tamoxifen.
\end{abstract}

\section{Materials and Methods}

The LMO5-E breast cancer cell line was used as a model for all experiments. Aldehyde dehydrogenase (ALDH) activity, clonogenic and mammosphere assays were performed to measure the effects of laminin on modulation of the stem cell subpopulation. Pluripotent gene expression was analyzed by reverse transcriptase-polymerase chain reaction. The involvement of the mitogen-activated protein kinase (MAPK)/ERK pathway was determined using specific inhibitors. The effects of laminin on the response to tamoxifen were determined and the involvement of $\alpha 6$ integrin was investigated.

\section{Results}

We found that pretreatment with laminin leads to a decrease in cells with the ability to form mammospheres that was accompanied by a decrease in ALDH activity. Moreover, exposure of mammospheres to laminin reduced the capacity to form secondary mammospheres and decreased the expression of Sox-2, Nanog, and Oct-4. We previously reported that 4-OHtamoxifen leads to an increase in the expression of these genes in LMO5-E cells. Treatment with signaling pathway inhibitors revealed that the MAPK/ERK pathway mediates the effects of laminin. Finally, laminin induced tamoxifen resistance in LM05-E cells through $\alpha 6$ integrin.

\section{Conclusion}

Our results suggest that the final number of cells with self-renewing capacity in estrogendependent breast tumors may result from the combined effects of endocrine treatment and microenvironmental cues.

\section{Introduction}

Seventy-five percent of women diagnosed with breast cancer have estrogen receptor (ER) and progesterone receptorpositive breast tumors [1,2]. Tamoxifen, which is a selective ER modulator, is the main 5-year adjuvant treatment for these patients [3]. However, one third of tamoxifen treated patients have recurrence within the first 15 years [4].
Key words

Laminin, Breast neoplasms, Estrogen receptor alpha, Stem cells, MAP kinase signaling system 
confers tamoxifen resistance through interaction with $\beta 1$ integrin [7].

There is consistent evidence suggesting that stem cells drive the growth and spread of breast tumors [12]. Moreover, several studies have shown that these cells are more resistant to conventional and endocrine therapy $[13,14]$. However, there is little evidence confirming the effects of the tumor microenvironment on regulation of the stem cell compartment. A few studies have shown involvement of extracellular matrix on the differentiation of embryonic stem cells in endoderm induction $[15,16]$. However, to the best of our knowledge, only one investigation of breast cancer has shown that the extracellular matrix leads to an increase in cells with stem cell properties [17].

We recently characterized the spontaneous M05 mouse mammary tumor that arose in a BALB/c mouse in our animal facility, and showed that it is estrogen dependent and tamoxifen sensitive in early passages, then progresses to endocrine resistance [18]. From this tumor, we generated a bicellular cell line, LM05-Mix, composed of both epithelial and fibroblastic cells that were subsequently separated to generate the epithelial LM05-E and fibroblastic LM05-F cell lines, respectively [19]. In LM05-E cells, we demonstrated that exposure to tamoxifen leads to an increase in cells with mammosphere forming capacity, which is in agreement with other studies [20,21].

The present study was conducted to further analyze regulation of the stem cell compartment in estrogen responsive breast cancer cell lines. In particular, we explored the effects of the extracellular matrix component laminin on LM05-E cells. We also studied the effects of this protein on the proportion of cells with stem cell properties. Our results suggest that for ER-positive breast cancer cells, exposure to the extracellular matrix component laminin leads to a decrease in cells with stem cell properties though the mitogen-activated protein kinase (MAPK)/ERK pathway, contrary to what is observed in response to tamoxifen. We also found that laminin produces resistance to tamoxifen induced cell death through $\alpha 6$ integrin. These findings suggest that, for ER-positive breast tumors, laminin modulates the final proportion of stem cells and the response to endocrine treatments such as tamoxifen.

\section{Materials and Methods}

\section{Cell culture}

The LM05-E cell lines were routinely maintained in growth medium consisting of Dulbecco's modified Eagle's medium
(DMEM)/F12 (Sigma-Aldrich, St. Louis, MO) supplemented with 10\% fetal calf serum (FCS; GenSA, Buenos Aires, Argentina) and gentamicin in a humidified $5 \% \mathrm{CO}_{2} /$ air atmosphere. Serial passages were conducted by treatment of $80 \%$ confluent monolayers with $0.25 \%$ trypsin (Invitrogen, Carlsbad, CA) and $0.02 \%$ EDTA in $\mathrm{Ca}^{2+}$-free and $\mathrm{Mg}^{2+}$-free phosphate buffered saline (PBS).

\section{Cell treatments}

To evaluate the effects of laminin, 500,000 cells were plated in $60 \mathrm{~mm}$ culture plates in growth medium. The next day, cells were washed twice with PBS and then treated in phenol red free DMEM/F12 with 1\% charcoal stripped FCS (chsFCS) and 17- $\beta$-estradiol (Sigma-Aldrich) at a final concentration of $10 \mathrm{nM}$. To test the role of laminin (Upstate Biotechnology, Lake Placid, NY), it was added at a final concentration of 2 $\mu \mathrm{g} / \mathrm{mL}$, after which samples were incubated for 48 hours. Mouse laminin purified from the Engelbreth Holm Swarm mouse tumor was used for all experiments. The laminin concentration was based on previous reports by other authors working with breast cancer cells [22] and our own preliminary observations. The following specific inhibitors were used during analyses: MAPK pathway inhibitor PD98059 $(10 \mu \mathrm{M}$, Calbiochem, Darmstadt, Germany), phosphoinositide 3-kinase (PI3K)/AKT pathway inhibitor LY294002 $(10 \mu \mathrm{M}$, Calbiochem). At least 1,000× stock solutions of the inhibitors were prepared in dimethyl sulfoxide (DMSO), and the equivalent dilutions of DMSO were used as controls. A 30-minute pretreatment with the inhibitors was conducted in each case before the 48-hour treatment.

We previously showed that treatment of LM05-E cells with 4-OH-tamoxifen leads to cell death based on a terminal deoxynucleotidyl transferase dUTP nick end labeling assay (In Situ Cell Death Detection Kit, Flourescein, Roche Applied Science, Bromma, Sweden) or propidium iodide (PI) exclusion $[7,19]$ in growth medium; therefore, the same protocol was used in this paper. Briefly, 35,000 cells were plated in eight well LabTek chamber slides (Nunc, Thermo Fisher Scientific, Roskilde, Denmark) in growth medium. The next day, samples were washed twice with phenol red free DMEM/F12, after which they were treated in the same culture medium supplemented with $1 \%$ chsFCS. 17- $\beta$-Estradiol (Sigma-Aldrich) was used at a final concentration of $10 \mathrm{nM}$, while 4-OH-tamoxifen (Sigma-Aldrich) was applied at $1 \mu \mathrm{M}$, as previously described [7]. Laminin $(2 \mu \mathrm{g} / \mathrm{mL})$ or bovine serum albumin (as a control) were also added at the time of treatment. To determine the involvement of $\alpha 6$ integrin, cells were pre-incubated for 15 minutes with the GoH3 monoclonal antibody $(0.15 \mu \mathrm{g} / \mathrm{mL}$, Santa Cruz Biotechnology [Santa Cruz, CA], purchased as azide and endotoxin free reagent) or with control pre-immune IgG (Santa Cruz 
Biotechnology, purchased as azide and endotoxin free reagent) before being plated in the chamber slides. The same procedure was used for the AIIB2 $\beta 1$ integrin blocking antibody $(0.15 \mu \mathrm{g} / \mathrm{mL}$, Aragen Bioscience, Morgan Hill, CA $)$ as previously described [7]. Antibodies were added to the culture medium when the treatments were started. After 48 hours of treatment, slides were washed with PBS, and cells were incubated for 1 minute in a PI solution $(50 \mu \mathrm{g} / \mathrm{mL}$ in PBS, Sigma-Aldrich), washed in PBS, and fixed with $4 \%$ formalin in PBS for 10 minutes. Nuclei were counterstained with 4',6-diamino-2-phenylindole (DAPI, Research Organics Inc., Cleveland, $\mathrm{OH})$, after which slides were mounted with Vectashield (Vector Laboratories, Burlingame, CA). Experiments were conducted in triplicate, and 10 random fields with an average of 200 cells / field were counted per well. The percentage of PI-positive cells relative to the total number of cells (stained with DAPI) was calculated as a measure of cell death and expressed as \% dead cells, as previously described [7]. Images were taken using a Nikon TE2000S (Tokyo, Japan) inverted fluorescent microscope and counted using the Image Pro Plus software (MediaCybernetics Inc., Silver Spring, MD).

\section{Mammosphere assays}

Single cell suspensions derived from the LM05-E cell line were plated in 6-well low attachment suspension culture plates (Greiner Bio-One, Koln, Germany) or 2\% agarose coated plates (for LM05-E cells) at a density of 10,000 viable cells $/ \mathrm{mL}$. Cells were grown in $2 \mathrm{~mL}$ serum-free media supplemented with B27 (Gemini Bioproducts, West Sacramento, $\mathrm{CA}$ ) and $20 \mathrm{ng} / \mathrm{mL}$ epidermal growth factor as previously described [14]. Mammospheres were counted after 5-8 days in culture using a Nikon Eclipse TE2000-S inverted microscope. To dissociate mammospheres to obtain single cell suspensions, spheres were centrifuged and resuspended in 100 $\mu \mathrm{L}$ of $0.25 \%$ trypsin and $0.53 \mathrm{mM}$ EDTA, then incubated for 5-10 minutes at $37^{\circ} \mathrm{C}$. Serum was added to stop the reaction, after which it was subjected to gentle pipetting and centrifugation. Finally, cells were resuspended in medium.

\section{Aldehyde dehydrogenase activity}

The Aldefluor kit (Stem Cell Technologies, Vancouver, Canada) was used to determine the percentage of aldehyde dehydrogenase (ALDH)-positive cells in the LM05-E cell line according to the manufacturer's instructions. Green fluorescence, which is produced as a result of ALDH activity, was analyzed by fluorescence-activated cell sorting analysis (PASIII, PARTEC, Munich, Germany).

\section{Clonogenic assays}

Single cell suspensions derived from the LM05-E cell line were plated in 24-well culture plates at a density of 200 cells/well. Cells were grown in $0.5 \mathrm{~mL}$ of growth medium for 7 days, after which the wells were washed and cells were fixed with methanol: acetic acid $(3: 1 \mathrm{v} / \mathrm{v})$ and then stained with crystal violet. Colonies of at least eight cells were counted using a binocular microscope.

\section{Reverse transcriptase-polymerase chain reaction}

RNA from subconfluent monolayers or from mammosphere cultures was prepared using TRIzol Reagent (Invitrogen Life Technologies). cDNA was prepared using an iScript cDNA synthesis kit (BioRad, Richmond, CA). PCR products were obtained using the mouse primers Nanog, Sox2, and Oct4 previously published by Zhang et al. [23].

\section{Western blot}

Subconfluent monolayers or mammosphere derived cells were pretreated with laminin for different lengths of time, after which cells were washed twice with ice-cold PBS, scraped with a Teflon scraper and finally lysed with $1 \%$ Triton X-100 in PBS. After protein determination, samples were denatured by boiling in sample buffer with $5 \% \beta$-mercaptoethanol and then run in 10\% sodium dodecyl sulfate polyacrylamide gel electrophoresis, with $50 \mu \mathrm{g}$ of protein loaded into each lane. Gels were subsequently blotted to Hybond-P membranes and incubated for 1 hour in blocking buffer containing 5\% skim milk and 0.1\% Tween-20 in PBS with p-ERK antibody (E-4: sc-7383, Santa Cruz BD, Dallas, TX), ERK1/2 antibody (MK1: sc-135900, Santa Cruz BD), or a6 integrin antibody (N-19: sc-6597, Santa Cruz BD) overnight at $4^{\circ} \mathrm{C}$, followed by 1 hour of incubation with a secondary antibody coupled to horseradish peroxidase. Samples were then subjected to chemiluminescence analysis, after which bands were digitalized with a Photo/Analyst Express System (Fotodyne Inc., Hartland, WI) and signal intensity was quantified with the Gel-Pro Analyzer software (Media Cybernetics, Rockville, MD).

\section{Statistical analysis}

Significant differences among assays were identified by the Student's $t$ test or one or two way ANOVA, followed by Bonferroni's comparisons test. A value of $p<0.05$ was considered significant. 
A
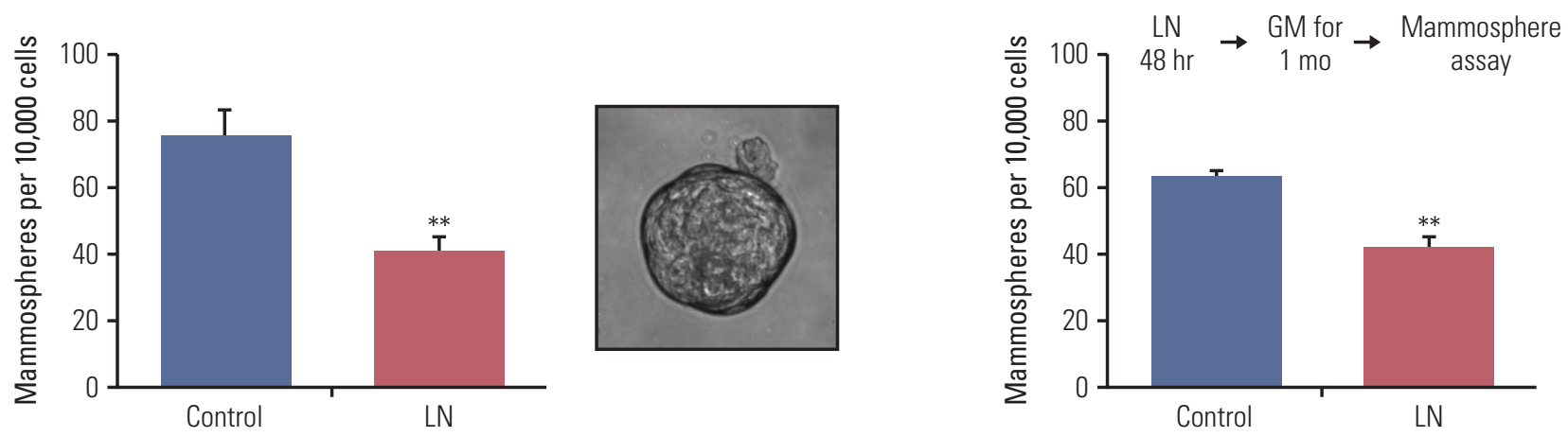

C
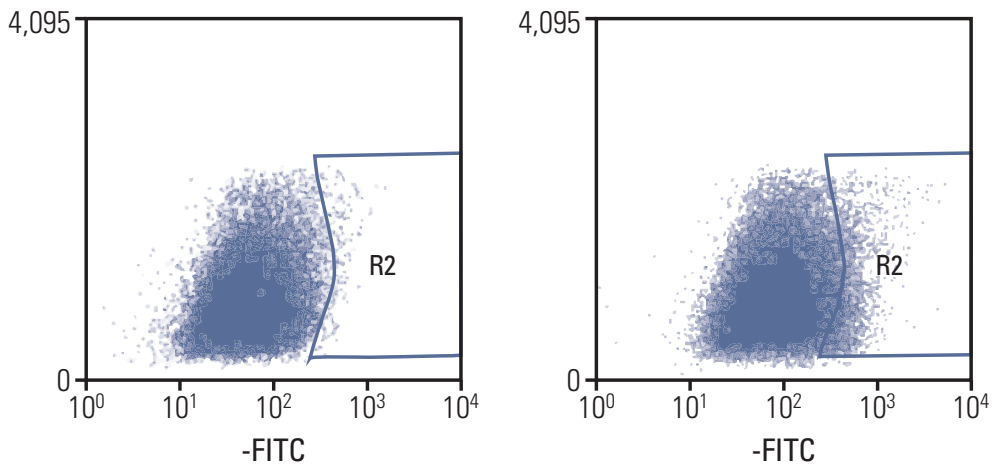

ALDH

Fig. 1. Laminin leads to a reduction in cells with mammosphere forming capacity and a decreased proportion of aldehyde dehydrogenase (ALDH)-positive cells. (A) LM05-E cells were treated for 48 hours in Dulbecco's modified Eagle's medium (DMEM) /F12 medium with 1\% charcoal stripped fetal calf serum (chsFCS) and $10 \mathrm{nM}$ estradiol supplemented with vehicle (control) or $2 \mu \mathrm{g} / \mathrm{mL}$ laminin (LN). Cells were then trypsinized, washed and plated on non-adherent 6-well plates at a density of 10,000 cells / $\mathrm{mL}$ in mammosphere medium as explained in the "Materials and Methods." The graphs show the number of mammospheres /10,000 cells after 6 days. A representative mammosphere is shown on the right. A significant reduction in mammosphere forming capacity was observed. (B) LM05-E cells were initially treated as in panel A, then maintained in growth medium (GM) for 1 month before conducting the mammosphere assays, as shown in the diagram above the graph. Even after 1 month in culture, a significant decrease in mammosphere forming capacity was observed for both cell lines. (C) Representative analysis of flow cytometries of cells treated (left) or untreated cells (right) with the ALDH inhibitor DEAB. Region 2 (R2) indicates cells that are considered positive in this assay. (Continued to the next page)

\section{Results}

\section{Laminin reduces the proportion of cells with stem cell properties}

The LM05-E breast cancer cell line is ER-positive and sensitive to tamoxifen [19]. We have previously shown that this cell line can form mammospheres and that this capacity is increased by tamoxifen [14]. In this study, the effects of laminin on the proportion of cells with stem cell properties were analyzed. To accomplish this, adherent cells were cultured in DMEM/F12 with 1\% chsFCS and estradiol $10 \mathrm{nM}$ supplemented with $2 \mu \mathrm{g} / \mathrm{mL}$ laminin or vehicle for 48 hours, after which the mammosphere-forming efficiency was assessed. After 48 hours, treatment was interrupted and cells were seeded into nonadherent plates under mammosphere forming conditions. As shown in Fig. 1A, laminin reduced 
D
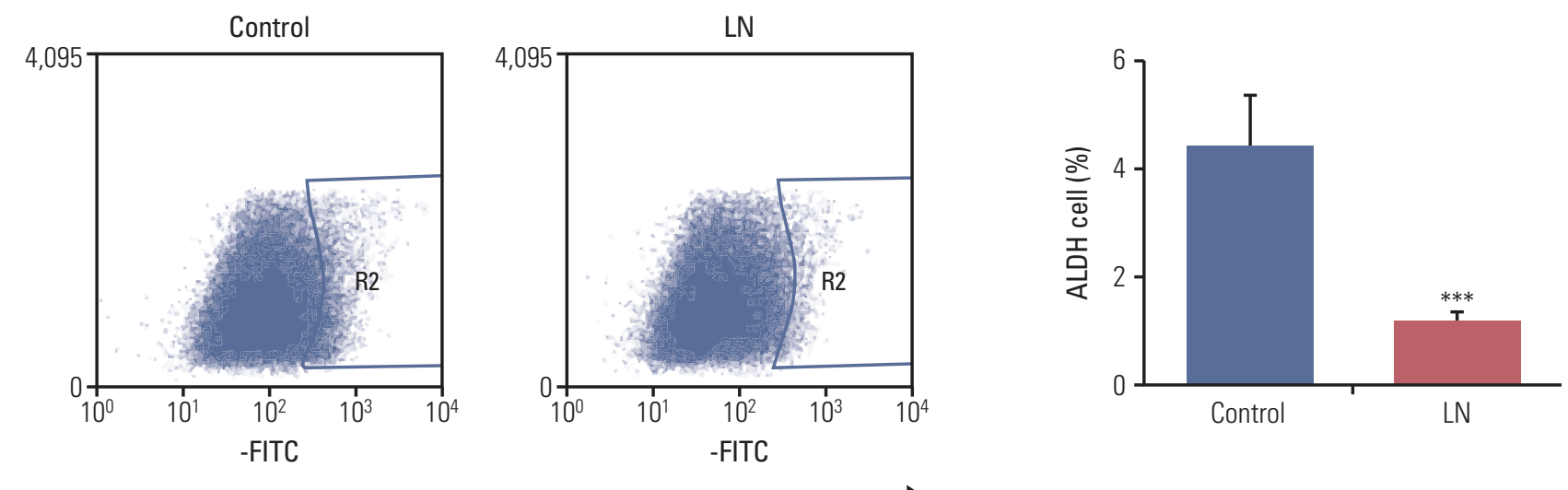

ALDH

Fig. 1. (Continued from the previous page) (D) LM05-E cells were treated for 48 hours in DMEM/F12 with 1\% chsFCS and 10 $\mathrm{nM}$ estradiol supplemented with vehicle (control) or $2 \mu \mathrm{g} / \mathrm{mL}$ LN. Cells were then trypsinized, washed and stained using the Aldefluor Kit (Stem Cell Technologies), after which they were analyzed by flow cytometry. Tubes without DEAB are shown and cells within region 2 are considered positive. (E) Quantification of the ALDH-positive cells is shown. One of at least three experiments is shown in each case $\left({ }^{* *} \mathrm{p}<0.01,{ }^{* * *} \mathrm{p}<0.001\right)$.

the mammosphere forming efficiency of LM05-E cells. Moreover, after treatment, cells were washed and then maintained under normal culture conditions for 1 month, at which time the mammosphere forming efficiency was assessed. The results observed after the additional month of culture were the same as those observed after the first 48-hour treatment, indicating that the effects of the initial treatment were sustained (Fig. 1B). No morphological changes were observed in the cells.

ALDH1 is a marker of stem cells [24]. To investigate the effects of laminin on the proportion of ALDH-positive cells, LM05-E cells were treated with laminin as explained above (for 48 hours on plastic), after which cells were processed as described in the materials and methods. Fig. 1C shows the representative flow cytometries of control cells to define the ALDH-positive cell regions. Fig. 1D shows the representative flow cytometries of cells treated with laminin and Fig. 1E displays the quantification of three independent experiments. The results indicate that laminin reduces the proportion of ALDH-positive cells, reinforcing the previous findings.

\section{Laminin reduces the clonogenic efficiency of LM05-E mammospheres}

We showed that laminin reduced the proportion of cells with stem cell properties. Next, the effects of laminin on the clonogenic efficiency of LM05-E mammospheres were studied. To accomplish this, mammospheres were cultured for

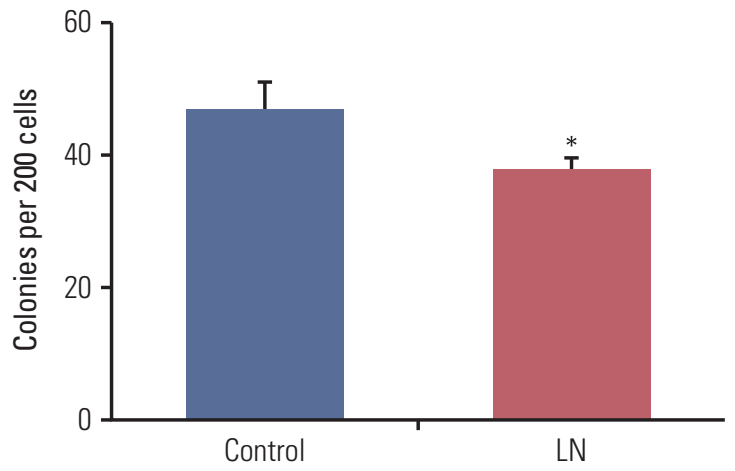

Fig. 2. Laminin (LN) reduces the clonogenic efficiency of mammospheres. Ten-day-old LM05-E mammospheres of LM05-E cells were treated for 48 hours with $2 \mu \mathrm{g} / \mathrm{mL} \mathrm{LN}$ or vehicle (control). Mammospheres were then dissociated and single cells were seeded at a concentration of 200 cells per well in 24-well plates in growth medium. Colonies of at least eight cells were counted after 7 days of culture. One of at least three experiments is shown $\left({ }^{*} \mathrm{p}<0.05\right)$.

10 days, then supplemented with laminin $(2 \mu \mathrm{g} / \mathrm{mL})$ or vehicle. Forty-eight hours later, mammospheres were disaggregated and seeded to study the clonogenic efficiency. As shown in Fig. 2, there was a significant decrease in the clonogenic efficiency of cells derived from mammospheres treated 
A

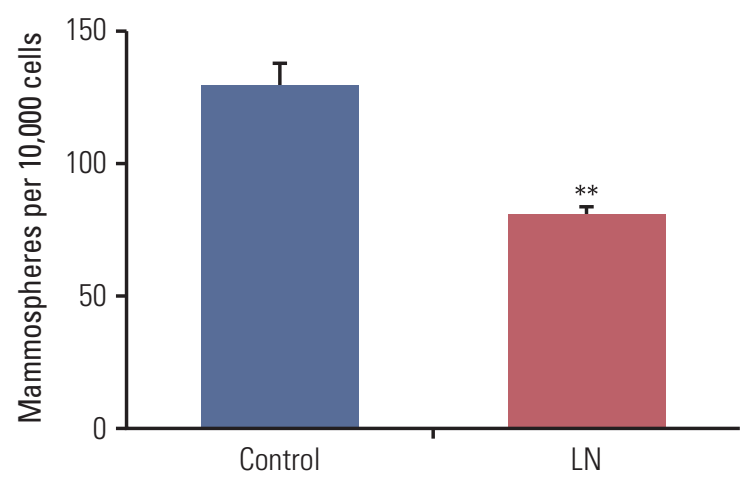

B

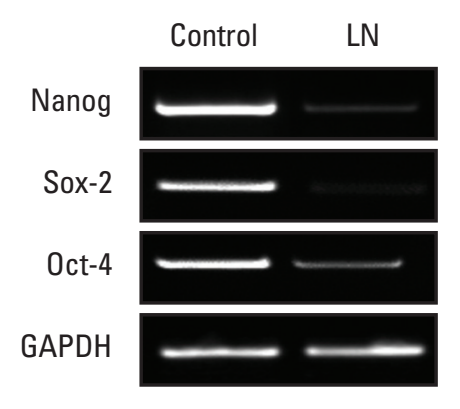

Fig. 3. Laminin (LN) induces differentiation of cells with stem cells properties. (A) Ten-day-old LM05-E mammospheres were supplemented for 48 hours with vehicle (control) or $2 \mu \mathrm{g} / \mathrm{mL} \mathrm{LN}$, then dissociated and seeded at a concentration of 10,000 cells per well in 6-well low attachment culture plates under mammosphere forming conditions. The number of mammospheres was quantified after 6 days. (B) LM05-E cells were pretreated with Dulbecco's modified Eagle's medium/F12, $1 \%$ charcoal stripped fetal calf serum supplemented with $10 \mathrm{nM}$ estradiol and $1 \mu \mathrm{M} 4-\mathrm{OH}$-tamoxifen or vehicle (control) for 5 days. Subsequently, cells were washed and cultured in growth medium for 1 week, after which they were treated with vehicle (control) or $2 \mu \mathrm{g} / \mathrm{mL}$ LN for 48 hours. Finally, RNA was extracted to investigate the gene expression of Nanog, Sox 2, Oct-4, and glyceraldehyde 3-phosphate dehydrogenase (GADPH) using reverse transcriptase-polymerase chain reaction. Treatment with LN led to a decrease in the expression levels of genes associated with self-renewal. One of at least three experiments is shown for each case $\left({ }^{* *} \mathrm{p}<0.01\right)$.

with laminin relative to the controls, suggesting that microenvironmental factors lead to cell differentiation and a reduction in the number of cells with stem cell properties.

\section{Laminin induces differentiation of cells with stem cell properties}

Considering that there is a reduction in the clonogenic potential of cells derived from mammospheres after treatment with laminin, we investigated whether these proteins induce cell differentiation. To accomplish this, mammospheres were treated with laminin as described above. Following treatment, mammospheres were disaggregated and seeded under mammosphere forming conditions and the number of secondary mammospheres was determined. As shown in Fig. 3A, for LM05-E, this matrix element reduced the number of secondary mammospheres, indicating that mammospheres contained less cells with self-renewing capacity after treatment. We next assessed if treatment with laminin modulated the expression of genes involved in stem cell self-renewal. We previously showed that a 5-day treatment with 4-OH-tamoxifen led to enrichment in cells with stem cell properties in the LM05-E cell line, as well as an increase in self renewal-associated gene expression [14]. In this context, we tested whether laminin could regulate the expression of genes associated with self-renewal after expo- sure to 4-OH-tamoxifen. To accomplish this, LM05-E cells were plated in cell culture dishes and treated with $4-\mathrm{OH}-$ tamoxifen for 5 days as previously described [14]. Cells were then washed and cultured in growth medium for one week. The adherent cells were subsequently treated as described above for 48 hours with laminin or vehicle, after which the RNA was extracted. The gene expression study revealed that laminin reduced Nanog, Sox2, and Oct-4 expression after tamoxifen treatment (Fig. 3B).

\section{The MAPK/ERK pathway mediates the effects of laminin over the stem cell population}

As shown above, laminin induced differentiation and reduced the proportion of cells with stem cell properties. We next proceeded to determine if either the PI3K/AKT or MAPK/ERK pathways were involved in this effect. To do so, LM05-E cells were subjected to a 30 minute pre-treatment with LY294002 (PI3K/AKT inhibitor) or PD98059 (MEK inhibitor). Next, laminin or vehicle was added to the cultures for 48 hours, after which mammosphere assays were conducted. As shown in Fig. 4A, treatment with the PI3K/AKT pathway inhibitor alone had an effect on LM05-E cells, indicating that cells with stem cell properties are dependent on this pathway. Fig. 4B shows that PD98059 inhibited the reduction in the number of mammospheres induced by the 


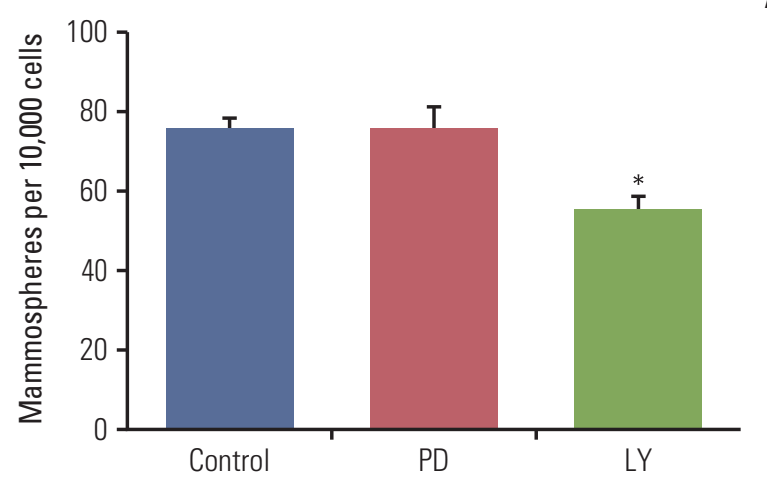

A

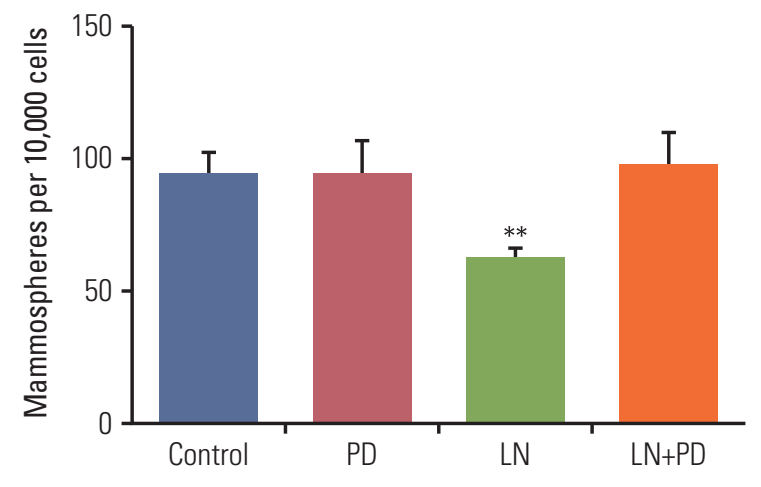

\section{C}

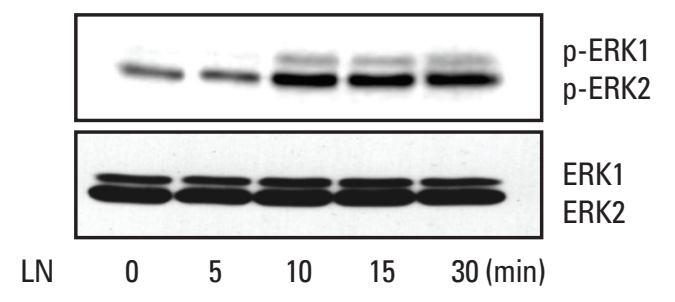

Fig. 4. The mitogen-activated protein kinase/ERK pathway mediates the effects of laminin (LN). (A) LM05-E cells were pretreated with $10 \mu \mathrm{M}$ PD98059 (PD), $10 \mu \mathrm{M} \mathrm{LY} 294002$ (LY), or vehicle (control) for 48 hours, after which mammosphere-forming capacity was assessed. (B) LM05-E cells were pre-treated with $10 \mu \mathrm{M}$ PD98059 (PD) or vehicle (control) for 30 minutes followed by $2 \mu \mathrm{g} / \mathrm{mL}$ LN for 48 hours, after which the mammosphere-forming capacity was analyzed. (C) LM05-E cells were treated with $2 \mu \mathrm{g} / \mathrm{mL} \mathrm{LN}$ for 5, 10, 15, and 30 minutes. Whole cell lysates were then prepared from treated LM05-E cells, resolved in $10 \%$ sodium dodecyl sulfate polyacrylamide gel electrophoresis and blotted with p-ERK and ERK antibodies. Activation of the ERK1/ 2 pathway was detected by LN treatment. One of at least three experiments is shown in each case $\left({ }^{*} \mathrm{p}<0.05,{ }^{* *} \mathrm{p}<0.01\right)$.

microenvironmental factor laminin in the LM05-E cell line. Western blot analysis of cells treated with laminin showed that this extracellular matrix component activates the MAPK/ ERK pathway in this cell line, as expected (Fig. 4C). These results indicate that the MAPK/ERK signaling pathway mediates the effects of laminin over the stem cell population in this ER-positive breast cancer cell line.

\section{Laminin induces tamoxifen resistance in LM05-E cells through $\alpha 6$ integrin}

We previously showed that fibronectin confers tamoxifen resistance to LM05-E cells through $\beta 1$ integrin [7]. Based on the effects of laminin on the stem cells population of the LM05-E line observed herein, we investigated whether laminin conferred tamoxifen resistance as well. To explore this possibility, cells were treated with 4-OH-tamoxifen for
48 hours in the presence of laminin $(2 \mu \mathrm{g} / \mathrm{mL})$. We found no increase in cell death under these conditions (Fig. 5A). To determine if the protective effects of laminin were mediated by $\alpha 6$ integrin, a well-established laminin receptor, western blot analysis of the protein extracts was conducted to confirm the expression of this specific integrin (Fig. 5B). Next, LM05E cells were pre-incubated with the $\mathrm{GoH} 3 \alpha 6$ integrin blocking antibody, then seeded on laminin. Treatment with GoH3 dramatically reduced the protective effects of laminin on tamoxifen-induced cell death (Fig. 5C). Interestingly, AIIB2, a $\beta 1$ integrin blocking antibody previously shown to revert fibronectin's protective effect [7], did not affect laminininduced endocrine resistance (Fig. 5D). Thus, our results show that, like fibronectin, laminin is able to induce tamoxifen resistance in LM05-E cells, and that in this case, $\alpha 6$ integrin mediates the protective effect. 

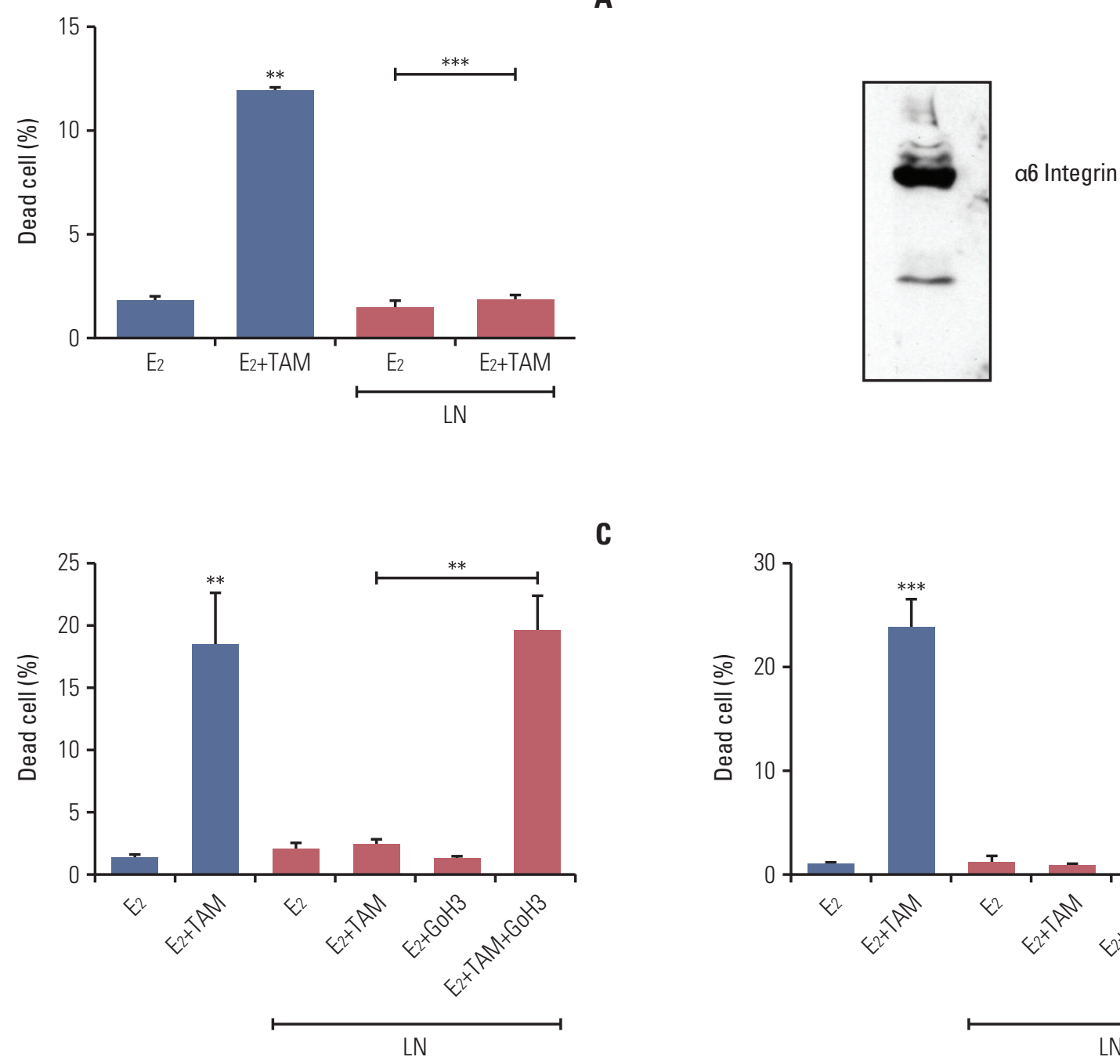

C

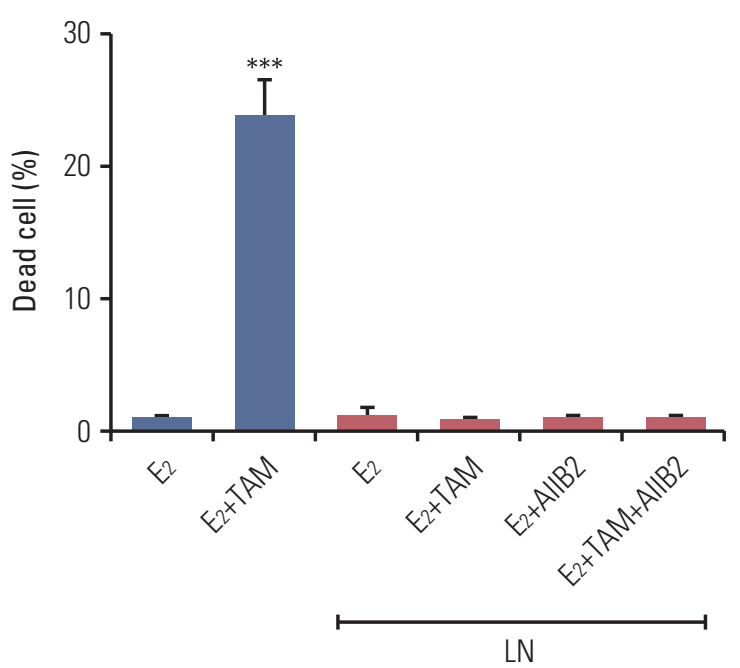

Fig. 5. Laminin (LN) induces tamoxifen resistance in LM05-E cells through integrin a6. (A) LM05-E cells were treated with 4-OH-tamoxifen (TAM) in the presence of laminin $(2 \mu \mathrm{g} / \mathrm{mL})$ for 48 hours, after which cell death was measured as described in the "Materials and Methods." (B) Whole cell lysates were prepared from LM05-E cells, resolved in 10\% sodium dodecyl sulfate polyacrylamide gel electrophoresis and blotted with a6 integrin antibody. (C) LM05-E cells were treated with TAM for 48 hours in the absence or presence of $\mathrm{LN}(2 \mu \mathrm{g} / \mathrm{mL})$ and/or the GoH3 $\alpha 6$ integrin blocking antibody, after which the percentage of dead cells was determined. (D) LM05-E cells were treated for 48 hours with TAM in the absence or presence of $\mathrm{LN}(2 \mu \mathrm{g} / \mathrm{mL})$ and / or the AIIB2 $\beta 1$ integrin blocking antibody and the percentage of dead cells was determined. One of at least three experiments is shown in each case $\left({ }^{* *} \mathrm{p}<0.01,{ }^{* * *} \mathrm{p}<0.001\right)$.

\section{Discussion}

In this study, we demonstrated that the microenvironmental factor laminin reduces the proportion of cells capable of forming mammospheres or with high ALDH1 activity through the MAPK/ERK pathway, while also inducing tamoxifen resistance through $\alpha 6$ integrin. It has been previ- ously determined that cells capable of forming mammospheres have stem cell properties [25], and that ALDH1 is a marker of normal and malignant breast cancer stem cells [24]. Based on these findings, we conclude that laminin reduces the proportion of cells with stem cell properties in the murine LM05-E cell line. Furthermore, we showed that these effects were abolished if an inhibitor of the MAPK/ERK pathways was used, indicating the involvement of this pathway in the 
previously described effect. We also demonstrated that treatment with laminin reduces the clonogenic potential of cells obtained from mammospheres and their capacity to form secondary mammospheres. Taken together, these results indicate that the number of cells with stem cells properties is diminished after treatment. Based on these findings, we assessed whether laminin was inducing differentiation by studying the expression of genes related to self-renewal. We previously showed that treatment of LM05-E cells with 4-OH-tamoxifen leads to an increase in the expression of genes associated with self-renewal and mammosphere forming capacity [14]. Therefore, we tested whether this effect could be counteracted by laminin. We found that this was the case, suggesting that the final number of stem cells in tamoxifen responsive breast cancer may result from the combined effects of treatment and the context.

To the best of our knowledge, only one other study has investigated the effects of extracellular matrix elements and cells with stem cell properties in breast cancer. Specifically, Saha et al. [17] studied the effects of laminin on neoplastic mouse mammary H605 cells. They found that microenvironmental elements led to an increase in the proportion of cells with stem cell properties using similar experimental approaches. The fact that these results are opposed to ours may be due to differences in experimental models. Specifically, H605 cells were obtained from primary cultures of a tumor derived from a 6-month-old Her2/neu-transgenic mouse [17]. However, similar results to ours have been reported in the context of extracellular matrix and stem cells during development. For example, Taylor-Weiner et al. [15] showed that laminin-111 is necessary for endoderm differentiation of embryonic stem cells. Moreover, other authors showed that $\beta 1$ integrin, which is the main receptor for extracellular matrix components, is also necessary for definitive endoderm differentiation [16]. Additionally, Prowse et al. [26] demonstrated that the MAPK/ERK and Rho-ROCK pathways are involved in signaling of extracellular matrix elements in embryonic stem cells. Other authors have reported that integrins and extracellular matrix elements are involved in the self-renewal, proliferation [27] and differentiation [28] of embryonic stem cells.

We previously showed that the extracellular matrix component fibronectin induces tamoxifen resistance in LM05-E cells [7]. In the present study, we demonstrated that laminin is able to induce endocrine resistance. In addition, we showed that tamoxifen treatment selects for cells with stem cell properties, leading to sustained enrichment of these cells in the LM05-E cell line [14]. Here, we show that cell lines with a high proportion of cells with stem cell properties after tamoxifen treatment respond to laminin by reducing this cell population, demonstrating the increased complexity of the relationship between cells and the microenvironment. Inter- estingly, we found that the effects of laminin are sustained over time, as indicated by cells exposed to laminin for 48 hours and then cultured in its absence for one month retaining a reduced number of mammosphere forming cells. Laminin has previously been shown to induce epigenetic changes in breast cancer cells $[29,30]$. Thus, in the context of our results we hypothesize that a mechanism of this type could be involved. Moreover, the effects of laminin on ER- $\alpha$ expression levels in the context of breast cancer stem cells have not been analyzed in this paper. Others have previously shown that laminin-1 increases the expression levels of ER- $\alpha$ in functionally normal primary mouse mammary epithelial cells [31]. Laminin induces differentiation of cells with stem cell properties, but also stimulates tamoxifen resistance. However, it remains to be determined if the reduction in the proportion of cells with stem cell properties induced by laminin modulates tamoxifen resistance in the LM05-E cell line or if it is an independent effect.

Our results suggest that the final number of cells with selfrenewing capacity in estrogen dependent breast tumors may result from the combined effect of endocrine treatment and microenvironmental cues. Further studies may reveal how strategies geared toward modulating the microenvironment may potentiate endocrine treatment in breast cancer and avoid enrichment in cells with stem cell properties.

\section{Conflicts of Interest}

Conflict of interest relevant to this article was not reported.

\section{Acknowledgments}

This work was supported by grants from MINCYT (PICT 20080325), Florencio Fiorini Foundation and the Instituto Nacional del Cáncer, Ministerio de Salud, to M.S. and a Conicet PIP grant (No. 11220110100557) to L.B.T. 


\section{References}

1. Harvey JM, Clark GM, Osborne CK, Allred DC. Estrogen receptor status by immunohistochemistry is superior to the ligand-binding assay for predicting response to adjuvant endocrine therapy in breast cancer. J Clin Oncol. 1999;17: 1474-81.

2. Graham JD, Yeates C, Balleine RL, Harvey SS, Milliken JS, Bilous AM, et al. Characterization of progesterone receptor A and B expression in human breast cancer. Cancer Res. 1995;55: 5063-8.

3. Sengupta S, Jordan VC. Selective estrogen modulators as an anticancer tool: mechanisms of efficiency and resistance. Adv Exp Med Biol. 2008;630:206-19.

4. Musgrove EA, Sutherland RL. Biological determinants of endocrine resistance in breast cancer. Nat Rev Cancer. 2009;9: 631-43.

5. Bissell MJ, Radisky D. Putting tumours in context. Nat Rev Cancer. 2001;1:46-54.

6. Tlsty T. Cancer: whispering sweet somethings. Nature. 2008;453:604-5.

7. Pontiggia O, Sampayo R, Raffo D, Motter A, Xu R, Bissell MJ, et al. The tumor microenvironment modulates tamoxifen resistance in breast cancer: a role for soluble stromal factors and fibronectin through beta1 integrin. Breast Cancer Res Treat. 2012;133:459-71.

8. Bergamaschi A, Tagliabue E, Sorlie T, Naume B, Triulzi T, Orlandi R, et al. Extracellular matrix signature identifies breast cancer subgroups with different clinical outcome. J Pathol. 2008;214:357-67.

9. Finak G, Bertos N, Pepin F, Sadekova S, Souleimanova M, Zhao $\mathrm{H}$, et al. Stromal gene expression predicts clinical outcome in breast cancer. Nat Med. 2008;14:518-27.

10. Helleman J, Jansen MP, Ruigrok-Ritstier K, van Staveren IL, Look MP, Meijer-van Gelder ME, et al. Association of an extracellular matrix gene cluster with breast cancer prognosis and endocrine therapy response. Clin Cancer Res. 2008;14: 5555-64.

11. Jansen MP, Foekens JA, van Staveren IL, Dirkzwager-Kiel MM, Ritstier K, Look MP, et al. Molecular classification of tamoxifen-resistant breast carcinomas by gene expression profiling. J Clin Oncol. 2005;23:732-40.

12. Cariati M, Naderi A, Brown JP, Smalley MJ, Pinder SE, Caldas C, et al. Alpha-6 integrin is necessary for the tumourigenicity of a stem cell-like subpopulation within the MCF7 breast cancer cell line. Int J Cancer. 2008;122:298-304.

13. Phillips TM, McBride WH, Pajonk F. The response of CD24 (-/low)/CD44+ breast cancer-initiating cells to radiation. J Natl Cancer Inst. 2006;98:1777-85.

14. Raffo D, Berardi DE, Pontiggia O, Todaro L, de Kier Joffe EB, Simian M. Tamoxifen selects for breast cancer cells with mammosphere forming capacity and increased growth rate. Breast Cancer Res Treat. 2013;142:537-48.

15. Taylor-Weiner H, Schwarzbauer JE, Engler AJ. Defined extracellular matrix components are necessary for definitive endoderm induction. Stem Cells. 2013;31:2084-94.
16. Liu J, He X, Corbett SA, Lowry SF, Graham AM, Fassler R, et al. Integrins are required for the differentiation of visceral endoderm. J Cell Sci. 2009;122(Pt 2):233-42.

17. Saha S, Lo PK, Duan X, Chen H, Wang Q. Breast tumour initiating cell fate is regulated by microenvironmental cues from an extracellular matrix. Integr Biol (Camb). 2012;4:897-904.

18. Simian M, Manzur T, Rodriguez V, de Kier Joffe EB, Klein S. A spontaneous estrogen dependent, tamoxifen sensitive mouse mammary tumor: a new model system to study hormone-responsiveness in immune competent mice. Breast Cancer Res Treat. 2009;113:1-8.

19. Pontiggia O, Rodriguez V, Fabris V, Raffo D, Bumaschny V, Fiszman $\mathrm{G}$, et al. Establishment of an in vitro estrogen-dependent mouse mammary tumor model: a new tool to understand estrogen responsiveness and development of tamoxifen resistance in the context of stromal-epithelial interactions. Breast Cancer Res Treat. 2009;116:247-55.

20. Piva M, Domenici G, Iriondo O, Rabano M, Simoes BM, Comaills $\mathrm{V}$, et al. Sox2 promotes tamoxifen resistance in breast cancer cells. EMBO Mol Med. 2014;6:66-79.

21. Simoes BM, Piva M, Iriondo O, Comaills V, Lopez-Ruiz JA, Zabalza I, et al. Effects of estrogen on the proportion of stem cells in the breast. Breast Cancer Res Treat. 2011;129:23-35.

22. Yang XH, Flores LM, Li Q, Zhou P, Xu F, Krop IE, et al. Disruption of laminin-integrin-CD151-focal adhesion kinase axis sensitizes breast cancer cells to ErbB2 antagonists. Cancer Res. 2010;70:2256-63.

23. Zhang L, Luo YB, Bou G, Kong QR, Huan YJ, Zhu J, et al. Overexpression Nanog activates pluripotent genes in porcine fetal fibroblasts and nuclear transfer embryos. Anat Rec (Hoboken). 2011;294:1809-17.

24. Ginestier C, Hur MH, Charafe-Jauffret E, Monville F, Dutcher $\mathrm{J}$, Brown M, et al. ALDH1 is a marker of normal and malignant human mammary stem cells and a predictor of poor clinical outcome. Cell Stem Cell. 2007;1:555-67.

25. Dontu G, Abdallah WM, Foley JM, Jackson KW, Clarke MF, Kawamura MJ, et al. In vitro propagation and transcriptional profiling of human mammary stem/progenitor cells. Genes Dev. 2003;17:1253-70.

26. Prowse AB, Chong F, Gray PP, Munro TP. Stem cell integrins: implications for ex-vivo culture and cellular therapies. Stem Cell Res. 2011;6:1-12.

27. Domogatskaya A, Rodin S, Boutaud A, Tryggvason K. Laminin- 511 but not -332 , -111, or -411 enables mouse embryonic stem cell self-renewal in vitro. Stem Cells. 2008;26: 2800-9.

28. Hunt GC, Singh P, Schwarzbauer JE. Endogenous production of fibronectin is required for self-renewal of cultured mouse embryonic stem cells. Exp Cell Res. 2012;318:1820-31.

29. Benton G, Crooke E, George J. Laminin-1 induces E-cadherin expression in 3-dimensional cultured breast cancer cells by inhibiting DNA methyltransferase 1 and reversing promoter methylation status. FASEB J. 2009;23:3884-95.

30. Pujuguet P, Radisky D, Levy D, Lacza C, Bissell MJ. Tricho- 
statin A inhibits beta-casein expression in mammary epithelial cells. J Cell Biochem. 2001;83:660-70.

31. Novaro V, Roskelley CD, Bissell MJ. Collagen-IV and laminin-
1 regulate estrogen receptor alpha expression and function in mouse mammary epithelial cells. J Cell Sci. 2003;116(Pt 14): 2975-86. 